\title{
ANFIS MODELS FOR SYNTHESIS OF OPEN SUPPORTED COPLANAR WAVEGUIDES
}

\author{
Sabri Kaya*, Kerim Guney† Celal Yildiz*, Mustafa Turkmen*
}

\begin{abstract}
Simple and accurate models based on adaptive-network-based fuzzy inference system (ANFIS) to compute the physical dimensions of open supported coplanar waveguides are presented. The ANFIS is a class of adaptive networks which are functionally equivalent to fuzzy inference systems. Four optimization algorithms, hybrid learning, simulated annealing, least-squares, and genetic, are used to determine optimally the design parameters of the ANFIS. When the performances of ANFIS models are compared with each other, the best results are obtained from the ANFIS models trained by the hybrid learning algorithm. The results of ANFIS are compared with the results of the conformal mapping technique, the rigorous spectral-domain hybrid mode analysis, the improved spectral domain approach, the synthesis formulas, a full-wave electromagnetic simulator IE3D, and experimental works realized in this study.
\end{abstract}

Key words: Supported coplanar waveguides, adaptive-network-based fuzzy inference system, synthesis, experiment

Received: May 10, 2010

Revised and accepted: November 20, 2013

\section{Introduction}

Coplanar waveguides (CPWs) have the advantages such as low dispersion, high flexibility in the design of characteristic impedance, and easy connection to the shunt lumped elements, or devices without using via holes [1-14]. CPWs and supported CPWs have received great attention due to their attractive features over the conventional microstrip lines in designing and manufacturing microwave integrated circuits (MICs) [1-14].

CPWs are often considered to have free space above and below the dielectric substrate. This configuration has not been found suitable for monolithic MICs

\footnotetext{
*Sabri Kaya, Celal Yildiz, Mustafa Turkmen

Erciyes University, Faculty of Engineering, Department of Electrical and Electronics Engineering, 38039, Kayseri, Turkey, E-mail: sabrikaya@erciyes.edu.tr, yildizc@erciyes.edu.tr, turkmen@erciyes.edu.tr

${ }^{\dagger}$ Kerim Guney

Nuh Naci Yazgan University, Faculty of Engineering, Department of Electrical and Electronics Engineering, Kayseri, Turkey, E-mail: kguney@erciyes.edu.tr
} 
(MMICs), where the substrate is typically thin and fragile. A solution is to mount the substrate directly on a conductor backed ground plane [5]. In this case, the ground plane will support the fragile substrate, thus increasing both the mechanical strength and the average power handling capability of the structure. However, it has been shown in $[6,7]$ that the ground plane backing introduces some undesirable effects on the CPW behaviour of the structure due to the presence of the microstrip mode. This mode can be suppressed by increasing the substrate thickness, but this is not always possible especially in MMIC applications where semiconductor substrates are usually thin. An alternative solution is to mount the semiconductor substrate on a low-permittivity material such as quartz then mount the entire assembly on a ground plane [6]. Another solution given in [8] is to grow a highquality GaAs layer on a Si substrate and then mount the entire assembly on a ground plane. In both cases $[6,8]$, the presence of supporting dielectric material under the main substrate will enhance the effect of the microstrip mode [9]. Hence, supported CPWs with infinitely thick supporting dielectric material under the main substrate have been proposed by Bedair and Wolff [10]. The thickness of the supporting dielectric material in supported CPW is large enough so that the effect of microstrip mode may be ignored.

Bedair and Wolff [10] have obtained the analytical formulas by using the conformal mapping technique (CMT) for computing the characteristic parameters of supported CPWs. In [10], the results of CMT were compared with results of rigorous spectral-domain hybrid mode analysis (RSDHMA). The quasi-TEM parameters of supported CPW configurations have been determined by using a numerically improved spectral domain approach (ISDA) [11]. The effective permittivities and characteristic impedances of the overlayed supported asymmetric CPWs were calculated in [12]. The artificial neural networks have been used to calculate the characteristic parameters of open supported CPWs (OS-CPWs) [13]. The formulas were proposed in [14] for synthesis of OS-CPWs.

Artificial neural networks (ANNs) [15] have been recently recognized as a fast and flexible tool in the analysis and design of electronic, electromagnetic and biomedical devices and circuits [13, 16-23]. They are efficient alternatives to conventional methods such as numerical modelling methods, analytical methods and empirical models. ANNs models have been used for computing the electroencenphalogarphy (EEG) forward solutions [16]. Feed forward neural networks have been proposed for solving the nonlinear forward problems in electrical capacitance tomography sensor systems [17]. Electromagnetically trained artificial neuralnetwork (EM-ANN) models have been developed for CPW components suitable for use in interactive MMIC design and optimization [18]. Various types of CPW structures have been analyzed by using the ANNs [19-23]. Fuzzy inference systems (FISs) have been proven to be strong tools and reliable models for tuning and design of microwave circuits [24-27]. Miraftab and Mansour have applied the FISs to the design of Chebyshev filters, elliptic filter, microstrip coupler, and microstrip filters [24-27]. In this work, a method based on adaptive-network-based fuzzy inference system (ANFIS) $[28,29]$ is presented to calculate accurately the physical dimensions of OS-CPWs for the required design specifications. ANFIS combines the benefits of ANNs and FISs in a single model. It has the advantages of expert knowledge of FISs and learning capability of ANNs. ANFIS is a class of adaptive 
Kaya S. et al.: ANFIS models for synthesis of open supported coplanar...

networks which are functionally equivalent to FISs. The FIS is a popular computing framework based on the concepts of fuzzy set theory, fuzzy if-then rules, and fuzzy reasoning. The ANFIS can simulate and analysis the mapping relation between the input and output data through a learning to determine optimal parameters of a given FIS. Fast and accurate learning, excellent explanation facilities in the form of semantically meaningful fuzzy rules, the ability to accommodate both data and existing expert knowledge about the problem, and good generalization capability features have made neuro-fuzzy systems popular in recent years [28-43]. In this paper, four different optimization algorithms, hybrid learning (HL) algorithm [28, 29], simulated annealing (SA) [44] algorithm, least-squares (LSQ) algorithm [45, 46], and genetic algorithm (GA) [47, 48], are used to train the ANFIS. These optimization algorithms are employed to obtain better performance and faster convergence with simpler structure. The validity and accuracy of the proposed ANFIS models are verified by comparing their results with the results of CMT [10], RSDHMA [10], ISDA [11], synthesis formulas [14], a full-wave electromagnetic simulator IE3D [49], and experimental works realized in this study.

\section{Adaptive - Network-Based Fuzzy Inference System (ANFIS)}

The ANFIS $[28,29]$ is a class of adaptive networks which are functionally equivalent to FISs. The selection of the FIS is the major concern in the design of an ANFIS. In this paper, the first-order Sugeno fuzzy model is used to generate fuzzy rules from a set of input-output data pairs. Among many FIS models, the Sugeno fuzzy model is the most widely applied one for its high interpretability and computational efficiency, and built-in optimal and adaptive techniques.

A typical architecture of ANFIS is shown in Fig. 1, in which a circle indicates a fixed node, whereas a square indicates an adaptive node. For simplicity to describe the procedure of the ANFIS, we assume that the FIS under consideration has two inputs $x$ and $y$ and one output $z$. For a first-order Sugeno fuzzy model, a common rule set with two fuzzy if-then rules can be written as

$$
\begin{aligned}
& \text { Rule 1: If } x \text { is } A_{1} \text { and } y \text { is } B_{1} \text {, then } z_{1}=p_{1} x+q_{1} y+r_{1} \text {, } \\
& \text { Rule 2: If } x \text { is } A_{2} \text { and } y \text { is } B_{2} \text {, then } z_{2}=p_{2} x+q_{2} y+r_{2}
\end{aligned}
$$

where $A_{i}$ and $B_{i}$ are the fuzzy sets in the antecedent, and $p_{i}, q_{i}$ and $r_{i}$ are the design parameters that are determined during the training process. As in Fig. 1, the ANFIS model has five layers. Each node in the first layer employs a node function given by

$$
\begin{array}{rlrl}
O_{i}^{1} & =\mu_{A_{i}}(x), & & i=1,2 \\
O_{i}^{1} & =\mu_{B_{i-2}}(y), & i=3,4
\end{array}
$$




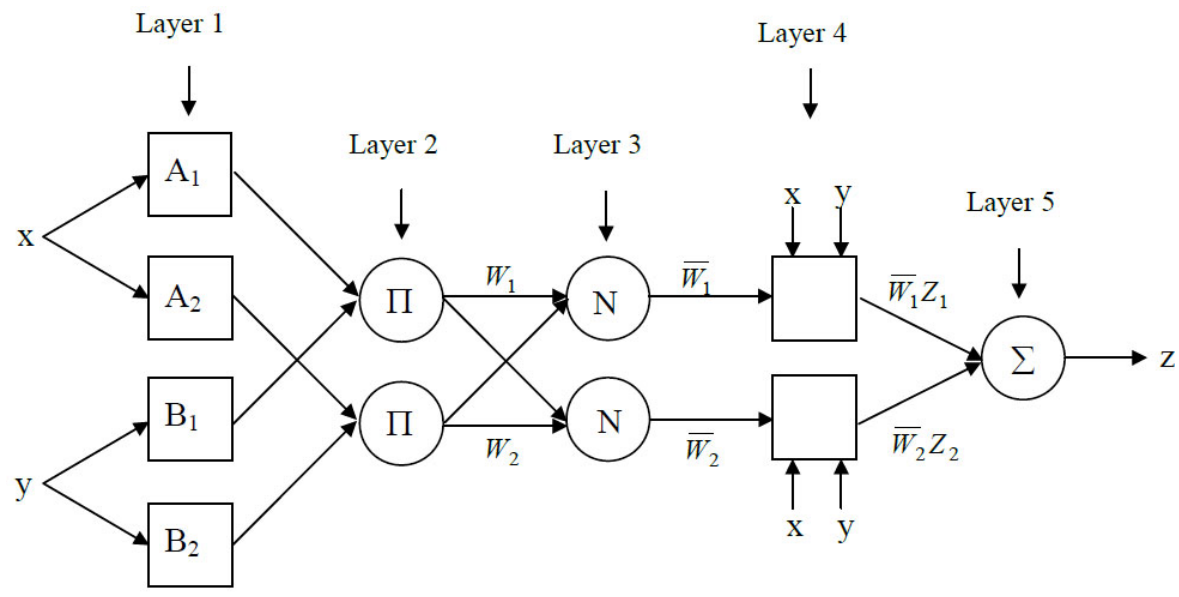

Fig. 1 Structure of an ANFIS model.

where $\mu_{A_{i}}(x)$ and $\mu_{B_{i-2}}(y)$ can adopt any fuzzy membership function (MF). In this paper, the following generalized bell (Gbell) MF is used.

$$
G_{b e l l}\left(x ; a_{i}, b_{i}, c_{i}\right)=\frac{1}{1+\left|\frac{x-c_{i}}{a_{i}}\right|^{2 b_{i}}}
$$

where $\left\{a_{i}, b_{i}, c_{i}\right\}$ is the parameter set that changes the shapes of the MF. Parameters in this layer are referred to as the premise parameters.

Each node in the second layer calculates the firing strength of a rule via multiplication:

$$
O_{i}^{2}=\omega_{i}=\mu_{A_{i}}(x) \mu_{B_{i}}(y), \quad i=1,2
$$

The $i$ th node in the third layer calculates the ratio of the $i$ th rule's firing strength to the sum of all rules' firing strengths:

$$
O_{i}^{3}=\overline{\omega_{i}}=\frac{\omega_{i}}{\omega_{1}+\omega_{2}}, \quad i=1,2
$$

where $\overline{\omega_{i}}$ is referred to as the normalized firing strengths.

In the fourth layer, each node has the following function:

$$
O_{i}^{4}=\overline{\omega_{i}} z_{i}=\overline{\omega_{i}}\left(p_{i} x+q_{i} y+r_{i}\right), \quad i=1,2
$$

where $\overline{\omega_{i}}$ is the output of layer 3 , and $\left\{p_{i}, q_{i}, r_{i}\right\}$ is the parameter set. Parameters in this layer are referred to as the consequent parameters.

The single node in the fifth layer computes the overall output as the summation of all incoming signals, which is expressed as:

$$
O_{1}^{5}=\sum_{i=1}^{2} \overline{\omega_{i}} z_{i}=\frac{\omega_{1} z_{1}+\omega_{2} z_{2}}{\omega_{1}+\omega_{2}}
$$


Kaya S. et al.: ANFIS models for synthesis of open supported coplanar...

The main objective of the ANFIS is to optimize the parameters of the fuzzy system parameters by applying an optimization algorithm using input-output data sets. The parameter optimization is done in a way such that the error measure between the target and the actual output is minimized. During the optimization process of the ANFIS, the premise parameters in the layer 1 and the consequent parameters in the layer 4 are tuned until the desired response of the FIS is achieved. In this paper, four different optimization algorithms, HL, SA, LSQ, and GA, are used to identify the parameters of ANFIS.

\section{ANFIS Models for the Synthesis of OS-CPWs}

The cross-section of an OS-CPW is illustrated in Fig. 2. In this figure, $s$ is the central strip width, $w$ is the slot width, $\varepsilon_{r 1}$ is the relative permittivity of supporting dielectric substrate, and $h$ is thickness of the main substrate with relative permittivity $\varepsilon_{r 2}$.

It is clear from the literature [10] that five parameters $\varepsilon_{r 1}, \varepsilon_{r 2}, h, s$, and $w$ are needed to determine the characteristic impedance of an OS-CPW. The first design step is the selection of a suitable main substrate $\left(\varepsilon_{r 2}, h\right)$ and supporting substrate $\left(\varepsilon_{r 1}\right)$ for an OS-CPW having a required characteristic impedance $Z_{0}$. Then, the physical dimensions $w$ and $s$ are determined. In this work, two simple and accurate ANFIS models are proposed for computing the slot and strip widths of OS-CPWs. The inputs of the first ANFIS model are $\varepsilon_{r 1}, \varepsilon_{r 2}, Z_{0}$, and $\mathrm{s} / h$, and the output is slot width $w$, as shown in Fig. 3(a). The first ANFIS model calculates the slot width $w$ for a given main substrate $\left(\varepsilon_{r 2}\right)$ and supporting substrate $\left(\varepsilon_{r 1}\right)$ and a required characteristic impedance $Z_{0}$ by choosing an appropriate normalized strip width $s / h$. The inputs of the second ANFIS model are $\varepsilon_{r 1}, \varepsilon_{r 2}, Z_{0}$, and $w / h$, and the output is strip width $\mathrm{s}$, as shown in Fig. 3(b). The second ANFIS model computes the strip width $s$ for a given main substrate $\left(\varepsilon_{r 2}\right)$ and supporting substrate $\left(\varepsilon_{r 1}\right)$ and a required characteristic impedance $Z_{0}$ by choosing an appropriate normalized slot width $w / h$.

The accuracy of a properly trained ANFIS depends on the accuracy and the effective representation of the data used for its training. A good collection of

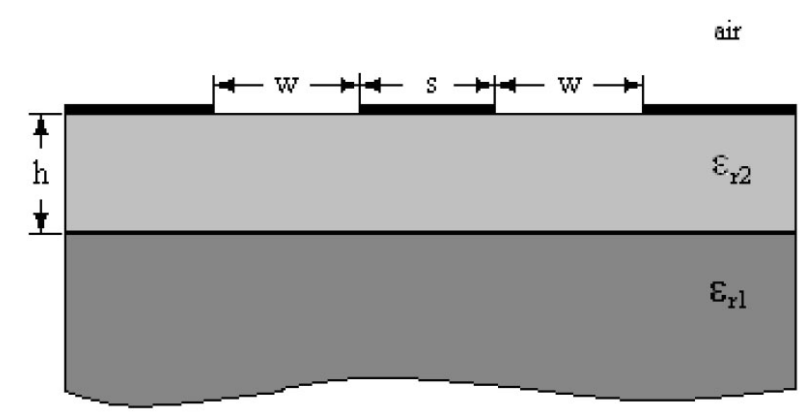

Fig. 2 Cross-section of an $O S-C P W$. 


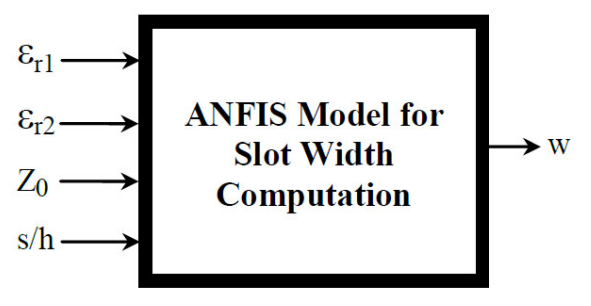

a)

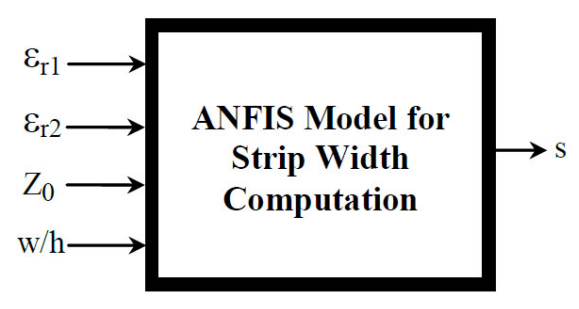

b)

Fig. 3 ANFIS models for the synthesis of OS-CPWs a) First ANFIS model and b) Second ANFIS model.

the training data, i.e., data which is well-distributed, sufficient, and accurately simulated, is the basic requirement to obtain an accurate model. If the training data sets are insufficient or do not cover all necessary representative features of the problem, it can cause large errors with testing data sets. If the training data sets are too much, this may cause overfitting and training may take quite a long time. There are two types of data generators for microwave applications. These data generators are measurement and simulation. The selection of a data generator depends on the application and the availability of the data generator. The training data sets used in this article have been obtained from the CMT [10]. 2732 data sets are used to train the ANFIS models. Data sets are in the range of $2 \leq \varepsilon_{r 1} \leq$ $10,10 \leq \varepsilon_{r 2} \leq 20,0.1 \leq s / h \leq 4,0.1 \leq w / h \leq 1,20 \mu \mathrm{m} \leq h \leq 2000 \mu m$, and the respective characteristic impedance is $19 \Omega \leq Z_{0} \leq 117 \Omega .562$ checking data sets obtained from CMT [10] are used to control the potential for the ANFIS models overfitting. The type of MFs for the input variables of ANFIS models is selected by using the checking errors. 971 data sets containing the results of CMT [10], RSDHMA [10], ISDA [11], synthesis formulas [14], IE3D [49], and experimental works realized in this study are used to test the ANFIS models. Checking and testing data sets are completely different from training data sets.

Training the ANFIS models with the use of an optimization algorithm to calculate slot widths $w$ or the strip widths $s$ involves presenting them sequentially and/or randomly with different sets $\left(\varepsilon_{r 1}, \varepsilon_{r 2}, Z_{0}\right.$, and $s / h$ or $\left.w / h\right)$ and corresponding physical dimensions $(w$ or $s)$. Differences between the target output and the actual outputs of the ANFIS are evaluated by the optimization algorithm. The adaptation is carried out after the presentation of each set $\left(\varepsilon_{r 1}, \varepsilon_{r 2}, Z_{0}, s / h\right.$ or 
Kaya S. et al.: ANFIS models for synthesis of open supported coplanar...

$w / h)$ until the calculation accuracy of the ANFIS is deemed satisfactory according to some criterion or when the maximum allowable number of epochs is reached.

The input and output data sets are scaled between 0 and 1 before training. The number of epoch is 1000 for training. The number of MFs is chosen as two for all input variables. The number of rules is then $16(2 \times 2 \times 2 \times 2=16)$. The type of MFs for the input variables is selected as the generalized bell. It is clear from Eq. (4) that the generalized bell MF is specified by three parameters. Therefore, ANFIS used here contains a total of 104 fitting parameters, of which $24(2 \times 3+2 \times 3+2 \times 3+2 \times 3=$ $24)$ are the premise parameters and $80(5 \times 16=80)$ are the consequent parameters.

It is well known that ANFIS has one output. For this reason, in this paper two separate ANFIS models with identical structure are used for calculating the slot and strip widths. Although the number of inputs, the number of MFs, and the types of MFs are the same for each ANFIS model, the values of premise and consequent parameters for each ANFIS model are different.

\section{Results and Discussion}

In this paper, two simple and accurate ANFIS models are proposed for OS-CPW synthesis. In order to check the accuracy of the method proposed in this paper, test results of ANFIS models are compared with the results of CMT [10], RSDHMA [10], ISDA [11], synthesis formulas [14], a full-wave electromagnetic simulator IE3D [49], and experimental works realized in this study.

The HL, SA, LSQ, and GA are used to determine optimally the design parameters of the ANFIS models. The training and test average percentage errors (APEs) of the first and second ANFIS models are given in Tab. I for computing the slot and strip widths of OS-CPWs. When the performances of ANFIS models are compared with each other, the best results are obtained from the models trained with the HL algorithm. Among the ANFIS models, the worst results are obtained from the models trained with the GA. The APEs values clearly show that the ANFIS models trained by HL algorithm can be used in computing the physical dimensions of OS-CPWs.

\begin{tabular}{|c|c|c|c|c|}
\hline \multirow{2}{*}{$\begin{array}{c}\text { Optimization } \\
\text { Algorithm }\end{array}$} & \multicolumn{2}{|c|}{$\begin{array}{c}\text { First ANFIS } \\
\text { Model }(w)\end{array}$} & \multicolumn{2}{c|}{$\begin{array}{c}\text { Second ANFIS } \\
\text { Model }(s)\end{array}$} \\
\cline { 2 - 5 } & Training & Test & Training & Test \\
\hline HL & 0.2005 & 0.2193 & 0.4322 & 0.4856 \\
\hline SA & 4.0259 & 4.1936 & 5.4603 & 5.4330 \\
\hline LSQ & 10.4860 & 10.9179 & 7.9451 & 8.0792 \\
\hline GA & 15.6200 & 17.0074 & 15.9932 & 16.0257 \\
\hline
\end{tabular}

Tab. I Training and test average percentage errors (\%) of ANFIS models.

In order to show clearly the validity and accuracy of the ANFIS models trained by HL algorithm, the results of the first and second ANFIS models are compared with the results of quasi-static analysis [10] in Figs. 4 and 5. Figs. 4 and 5, respectively, illustrate the quasi-static analysis [10] contours, the slot width $w$ results 
obtained by first ANFIS model and the strip width $s$ results obtained by second ANFIS model for OS-CPWs with GaAs $\left(\varepsilon_{r 2}=12.9\right.$ and $\left.h=200 \mu \mathrm{m}\right)$ supported by quartz $\left(\varepsilon_{r 1}=3.78\right)$ and a required characteristic impedance. It is apparent from Figs. 4 and 5 that there is a very good agreement between the results of quasi-static analysis [10] and the ANFIS models. Similar contours are achieved for the different dielectric substrate materials $\left(2 \leq \varepsilon_{r 1} \leq 10\right.$ and $\left.10 \leq \varepsilon_{r 2} \leq 20\right)$, but they are not given here to avoid repetition.

The characteristic impedances computed by using the results of ANFIS models trained by the HL algorithm are compared with those of quasi-static analysis [10] for OS-CPWs with hypothetical substrate $\left(\varepsilon_{r 2}=20\right.$ and $\left.h=250 \mu \mathrm{m}\right)$ supported by alumina $\left(\varepsilon_{r 1}=10\right)$ in Fig. 6. In this figure, the characteristic impedance results are plotted with respect to the shape ratio $(s+w) / h$ for $s / h=0.3,1,2.5$, and 4. It can be seen from Fig. 6 that the results of the ANFIS models are in very good agreement with the results of quasi-static analysis [10]. It is also evident from this figure that there is a very good self-consistent agreement between the first and second ANFIS models.

In order to make a further comparison, the given geometrical values, the geometrical values calculated from the first and second ANFIS models, and the characteristic impedances determined by using the geometrical values calculated by the ANFIS models trained by the HL algorithm, CMT [10], RSDHMA [10], and ISDA [11] are listed in Tabs. II-IV for three different cases of OS-CPWs. These cases are: hypothetical substrate $\left(\varepsilon_{r 2}=20\right)$ supported by alumina $\left(\varepsilon_{r 1}=10\right)$, GaAs $\left(\varepsilon_{r 2}\right.$ $=12.9)$ supported by quartz $\left(\varepsilon_{r 1}=3.78\right)$, and GaAs $\left(\varepsilon_{r 2}=12.9\right)$ supported by alumina $\left(\varepsilon_{r 1}=10\right)$. The results of the synthesis formulas [14] in the literature are also given in these tables for comparison. In Tabs. II-IV, $w^{\prime}$ and $s$ ' represent the given geometrical values of slot and strip widths of OS-CPWs, respectively. $Z_{0}\left(w^{\prime}\right.$, $\left.s^{\prime}\right)$ represents the characteristic impedance values obtained from CMT, RSDHMA, and ISDA by using the given geometrical values $w^{\prime}$ and $s^{\prime} . w^{*}$ and $s^{*}$ represent the slot and strip widths obtained from the first and second synthesis formulas [14] by using the $s^{\prime}$ and $w^{\prime}$, respectively. $Z_{0}\left(w^{*}, s^{\prime}\right)$ and $Z_{0}\left(w^{\prime}, s^{*}\right)$ are the final-check quasi-static analysis results calculated by using the $w^{*}$ and $s^{*}$ values, respectively. $w$ and $s$ represent the slot and strip widths obtained from the first and second ANFIS models by using the $s^{\prime}$ and $w^{\prime}$, respectively. $Z_{0}\left(w, s^{\prime}\right)$ and $Z_{0}\left(w^{\prime}, s\right)$ are the final-check quasi-static analysis results calculated by using the $w$ and $s$ values, respectively. As it can be seen from Tabs. II-IV, there is a very good agreement between the geometrical values ( $w$ and $s$ ) calculated by the ANFIS models and the given geometrical values ( $w^{\prime}$ and $\left.s^{\prime}\right)$. This very good agreement supports the validity of the proposed ANFIS models. The accurate determination of the geometrical values $(w$ and $s$ ) by using ANFIS models leads to good accuracy in the calculation of the characteristic impedances. It is also clear from Tabs. II-IV that ANFIS models provide more accurate results than the synthesis formulas presented in [14].

In this paper, five different OS-CPWs are fabricated on RT/duroid laminates $\left(\varepsilon_{r 1}=6.15, \varepsilon_{r 2}=10.2\right.$, and $\left.h=1270 \mu \mathrm{m}\right)$ by using the printed circuit board (PCB) excavation technique. The characteristic impedances of these OS-CPWs are calculated from the measured $S$-parameters for $2 \mathrm{GHz}$ [3]. We also calculated the characteristic impedances by using a full-wave electromagnetic simulator IE3D 
Kaya S. et al.: ANFIS models for synthesis of open supported coplanar...

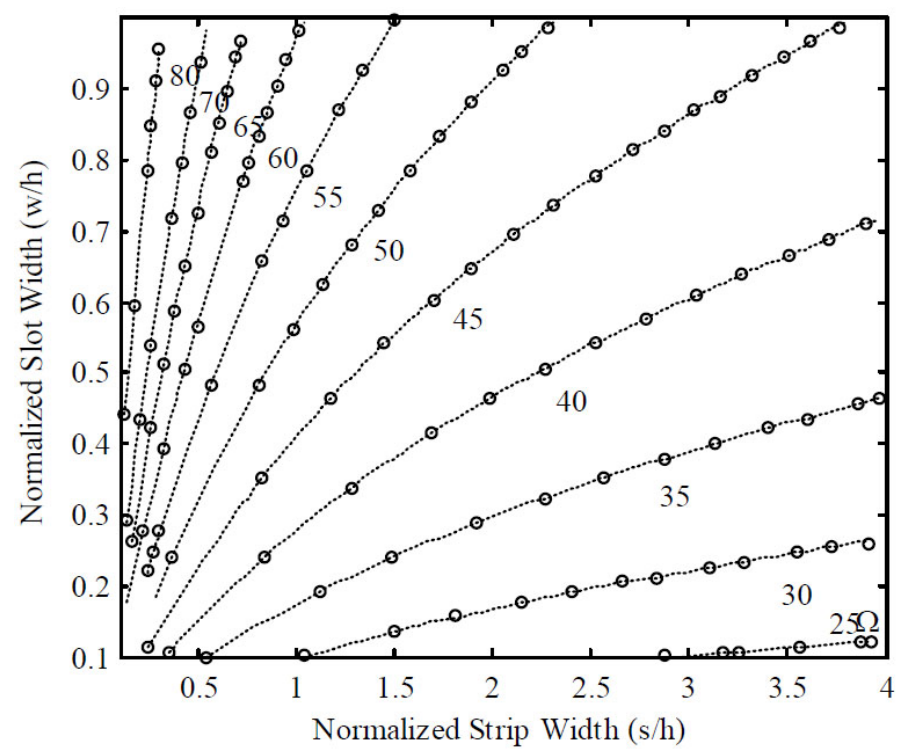

Fig. 4 Comparison of the slot width $(w)$ results obtained by using the first ANFIS model and the quasi-static analysis [10] contours for OS-CPWs $\left(\varepsilon_{r 1}=3.78, \varepsilon_{r 2}=\right.$ 12.9 , and $h=200 \mu \mathrm{m})$.

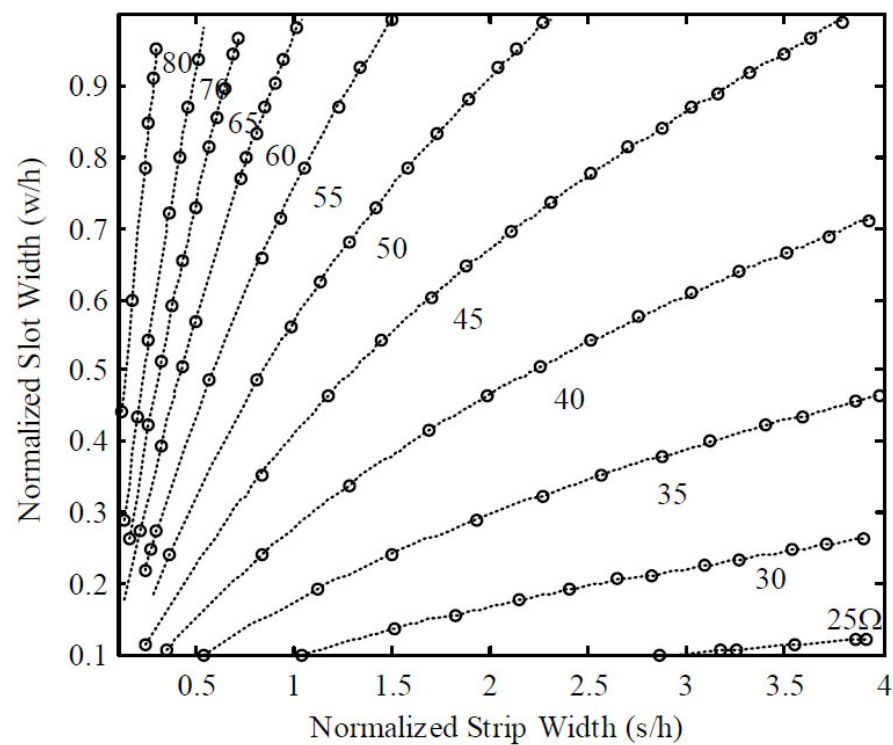

Fig. 5 Comparison of the strip width (s) results obtained by using the second ANFIS model and the quasi-static analysis [10] contours for OS-CPWs $\left(\varepsilon_{r 1}=3.78, \varepsilon_{r 2}=\right.$ 12.9 , and $h=200 \mu m)$. 


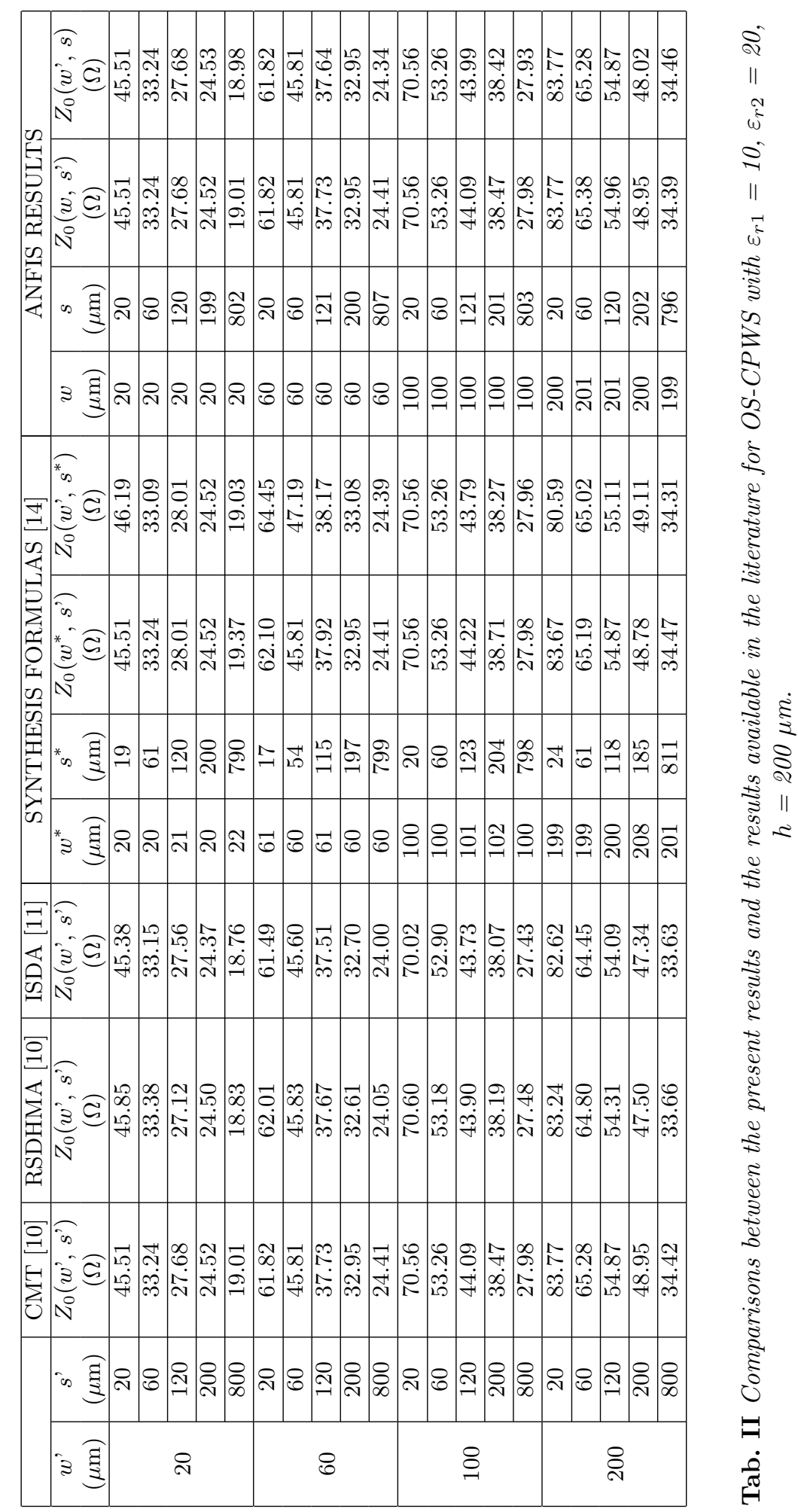


Kaya S. et al.: ANFIS models for synthesis of open supported coplanar...

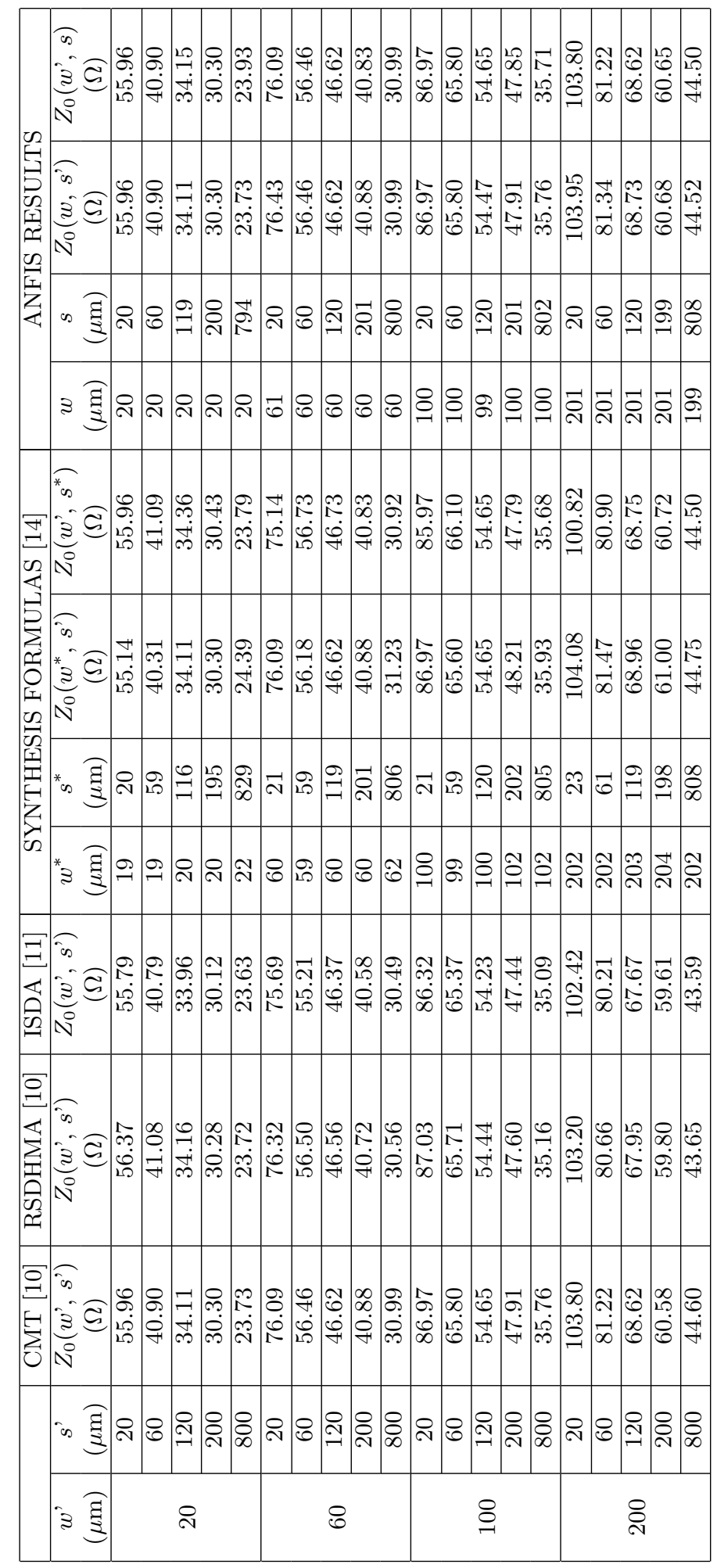

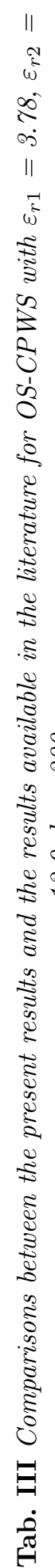




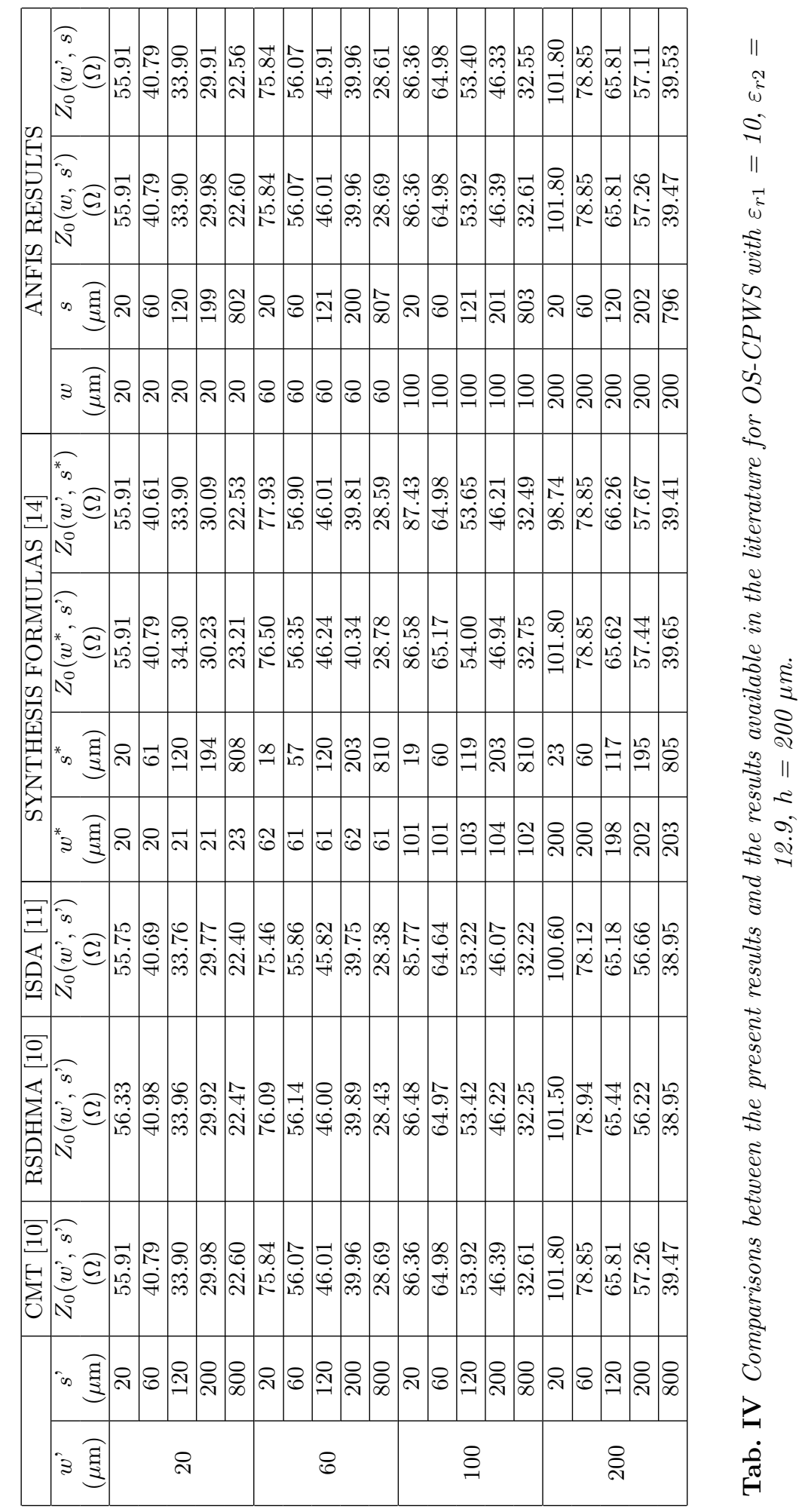


Kaya S. et al.: ANFIS models for synthesis of open supported coplanar...

[49]. In Tab. V, the results of the ANFIS models trained by the HL algorithm are compared with the results of CMT [10], synthesis formulas [14], IE3D [49], and experimental works realized in this study. As it can be seen from Tab. V, a good agreement is obtained between the theoretical and experimental results.

A prominent advantage of ANFIS computation is that, after proper training, an ANFIS completely bypasses the repeated use of complex iterative processes for new cases presented to it. Thus, the ANFIS is very fast after training. The ANFIS structure can be implemented in real time by using state-of-the art hardware devices, such as FPGAs (Field Programmable Gate Array). In this way, the computation time of the system is limited only by the response time of the FPGA, which is in the order of a few microseconds.

\section{Conclusion}

In this paper, simple and accurate ANFIS models are presented for computing the physical dimensions of OS-CPWs. The HL, SA, LSQ, and GA are used to identify the parameters of ANFIS. The best result is obtained from the ANFIS trained by HL algorithm. The results of ANFIS are in good agreement with the measurements, and better accuracy with respect to the previous synthesis formulas is obtained. The ANFIS models allow the designers to determine the physical dimensions of OSCPWs for the required design specifications by a very simple and convenient way,

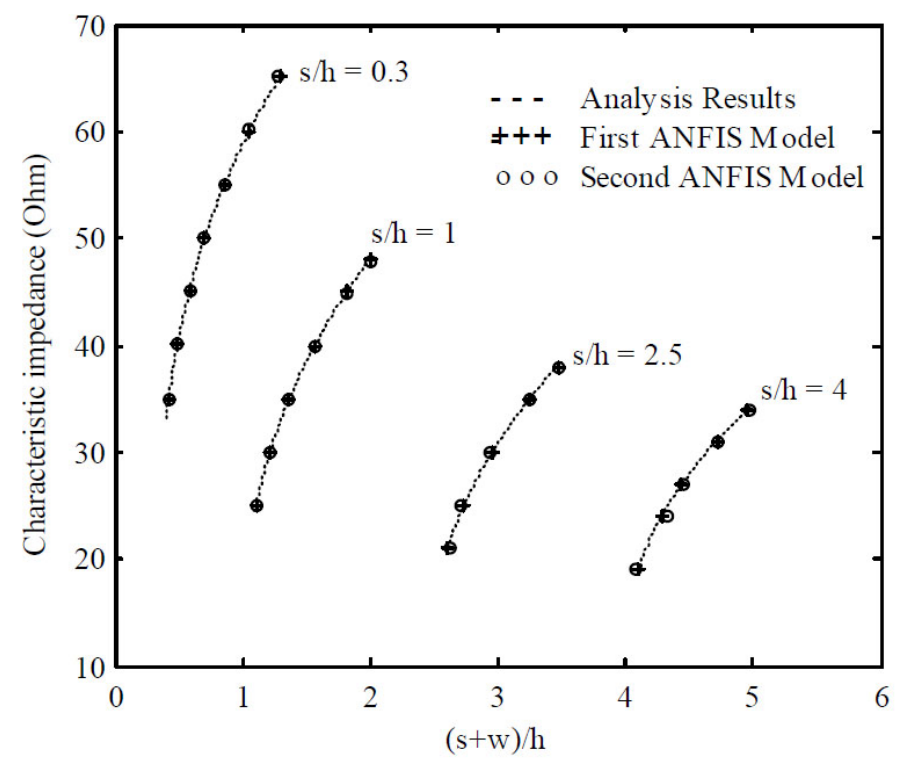

Fig. 6 Comparisons of the characteristic impedances calculated by using the result of the first ANFIS model for a given s; the result of the second ANFIS model for a given $w$; and the quasi-static analysis [10] for OS-CPWs $\left(\varepsilon_{r 1}=10, \varepsilon_{r 2}=20\right.$, and $h=250 \mu \mathrm{m})$. 


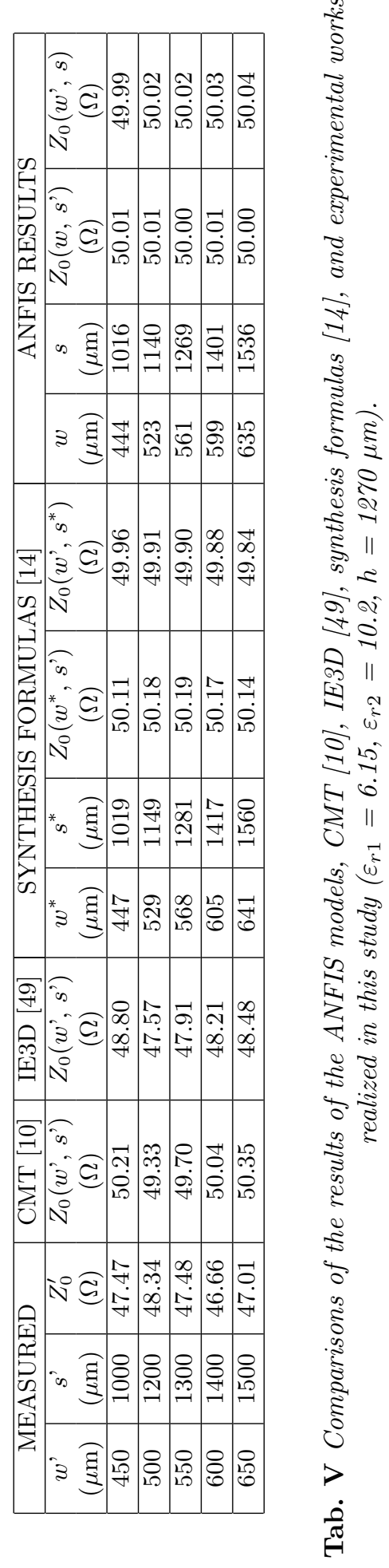


Kaya S. et al.: ANFIS models for synthesis of open supported coplanar...

rather than by the iteration approach of applying analysis technique. ANFIS is a very powerful approach for building complex and nonlinear relationship between a set of input and output data. The high-speed real-time computation feature of the ANFIS recommends its use in microwave CAD programs.

\section{Acknowledgment}

The work described in this paper is supported by The Scientific and Technological Research Council of Turkey (TUBITAK), (Project No: 107E258).

\section{References}

[1] Wen C. P.: Coplanar waveguide: A surface transmission line suitable for nonreciprocal gyromagnetic device applications. IEEE Trans. Microwave Theory and Techniques, 17, 1969, pp. 1087-1090.

[2] Veyres C., Hanna V. F.: Extension of application of conformal mapping techniques to coplanar lines with finite dimensions. International Journal of Electronics, 48, 1980, pp. 47-56.

[3] Simons R. N.: Coplanar Waveguide Circuits, Components, and Systems, Wiley, New York, 2001.

[4] Wolff I.: Coplanar Microwave Integrated Circuits, John Wiley \& Sons, New Jersey, 2006.

[5] Ghione G., Naldi C.: Parameters of coplanar waveguides with lower ground plane. Electronic Lett., 19, 1993, pp. 734-735.

[6] Jackson R. W.: Considerations in the use of coplanar waveguide for millimeter-wave integrated circuits. IEEE Trans. Microwave Theory and Techniques, 34, 1986, pp. 1450-1456.

[7] Riaziat M., Bandy S., Zdasiuk G.: Coplanar waveguides for MMICs. Microwave Journal, 30, 1987, 125, 128, 130.

[8] Aksun M. I., Morkoc H.: GaAs on $\mathrm{Si}$ as a substrate for microwave and millimeter-wave monolithic integration. IEEE Trans. Microwave Theory and Techniques, 36, 1988, pp. 160162.

[9] Shigesawa H., Tsuji M., Conductor-backed slot line and coplanar waveguide: Dangers and full-wave analysis. IEEE MTT-S Dig, 1988, pp. pp. 199-202.

[10] Bedair S. S., Wolff I.: Fast, accurate and simple approximate analytic formulas for calculating the parameters of supported coplanar waveguides for (M)MIC's. IEEE Trans. Microwave Theory and Techniques, 40, 1992, pp. 41-48.

[11] Cheng K. K. M., Robertson I. D.: Numerically efficient spectral domain approach to the quasi-TEM analysis of supported coplanar waveguide structures. IEEE Trans. Microwave Theory and Techniques, 42, 1994, pp. 1958-1965.

[12] Gorur A., Karpuz C., Alkan M, Quasistatic TEM characteristics of overlayed supported asymmetric coplanar waveguides. Int. J. RF Microwave and Millimeter-Wave ComputerAided Engineering, 6, 1996, pp. 297-304.

[13] Yildiz C., Guney K., Turkmen M., Kaya S.: Neural models for quasi-static analysis of conventional and supported coplanar waveguides. AEU-Int. J. Electron. Commun., 61, 2007, pp. 521-527.

[14] Kaya S., Guney K., Yildiz C., Turkmen M.: New and accurate synthesis formulas for open supported coplanar waveguides. Microwave Opt. Technol. Lett., 52, 2010, pp. 262-269.

[15] Haykin S.: Neural Networks: A Comprehensive Foundation. Macmillan College Publishing Comp., New York, 1994.

[16] Sun M, Sclabassi R. J.: The forward EEG solutions can be computed using artificial neural networks. IEEE Trans. Biomedical Engineering, 47, 2000, pp. 1044-1050. 


\section{Neural Network World 6/13, 553-569}

[17] Marashdeh Q., Warsito W., Fan L.-S., Teixeira F. L.: Nonlinear forward problem solution for electrical capacitance tomography using feed forward neural network. IEEE Sensors Journal, 6, 2006, pp. 441-449.

[18] Watson P. M., Gupta K. C.: Design and optimization of CPW circuits using EM-ANN models for CPW components. IEEE Microwave Theory and Techniques, 45, 1997, pp. 2515-2523.

[19] Zhang Q. J., Gupta K. C.: Neural Networks for RF and Microwave Design. Artech House, Boston MA, 2000.

[20] Christodoulou C.G., Georgiopoulos M.: Application of Neural Networks in Electromagnetics. Artech House, MA, 2001.

[21] Yildiz C., Turkmen M.: A CAD approach based on artificial neural networks for shielded multilayered coplanar waveguides. AEU-International Journal of Electronics and Communications, 58, 2004, pp. 284-292.

[22] Guney K., Yildiz C., Kaya S., Turkmen M.: Neural models for the broadside-coupled Vshaped microshield coplanar waveguides. International Journal of Infrared and Millimeter Waves, 27, 2006, pp. 1241-1255.

[23] Guney K., Yildiz C., Kaya S., Turkmen M.: Neural models for the V-shaped conductorbacked coplanar waveguides. Microwave Opt. Technol. Lett., 49, 2007, pp. 1294-1299.

[24] Miraftab V., Mansour R. R.: Computer-aided tuning of microwave filters using fuzzy logic, Trans. Microwave Theory and Techniques. 50, 2002, pp. 2781-2788.

[25] Miraftab V., Mansour R. R.: A robust fuzzy-logic technique for computer-aided diagnosis of microwave filters. IEEE Trans. Microwave Theory and Techniques, 52, 2004, pp. 450-456.

[26] Miraftab V., Mansour R. R.: EM-based microwave circuit design using fuzzy logic techniques. IEE Proc.-Microw. Antennas Propag., 153, 2006, pp. 495-501.

[27] Miraftab V., Mansour R. R.: Fully automated RF/Microwave filter tuning by extracting human experience using fuzzy controllers. IEEE Trans. Circuits and Systems, 55, 2008, pp. $1357-1367$.

[28] Jang J.-S.R.: ANFIS: Adaptive-network-based fuzzy inference system. IEEE Trans. Syst. Man Cybern., 23, 1993, pp. 665-685.

[29] Jang J.-S. R., Sun C. T., Mizutani E.: Neuro-Fuzzy and Soft Computing: A Computational Approach to Learning and Machine Intelligence. Prentice-Hall, New Jersey, 1997.

[30] Guney K., Sarikaya N.: Adaptive neuro-fuzzy inference system for the input resistance computation of rectangular microstrip antennas with thin and thick substrates. J. Electromagn. Waves Applicat., 18, 2004, pp. 23-39.

[31] Guney K., Sarikaya N.: Computation of resonant frequency for equilateral triangular microstrip antennas using the adaptive neuro-fuzzy inference system. Int. J. RF Microwave Computer-Aided Engineering, 14, 2004, pp. 134-143.

[32] Rahouyi E. B., Hinojosa J., Garrigos J.: Neuro-fuzzy inference modeling techniques for microwave components. IEEE Microwave and Wireless Components Lett. 16, 2006, pp. 7274.

[33] Hinojosa J., Dome'nech-Asensi G.: Space-mapped neuro-fuzzy optimization for microwave device modeling. Microwave Opt. Technol. Lett., 49, 2007, pp. 1328-1334.

[34] Hinojosa J., Dome'nech-Asensi G., Martı'nez-Alajarı'n J.: Development of VHDL-AMS neuro-fuzzy behavioral models for $\mathrm{RF} /$ microwave passive components. Int. J. RF Microwave Computer-Aided Engineering, 17, 2007, pp. 335-344.

[35] Brown M., Haris C., Neuro-Fuzzy Adaptive Modeling and Control, Prentice-Hall, Englewood Cliffs, NJ, 1994.

[36] Constantin V. A.: Fuzzy Logic and Neuro-Fuzzy Applications Explained, Prentice-Hall, Englewood Cliffs, NJ, 1995.

[37] Lin C. T., Lee C. S. G., Neural Fuzzy Systems: A Neuro-Fuzzy Synergism to Intelligent Systems, Prentice- Hall, Upper Saddle River, NJ, 1996. 
Kaya S. et al.: ANFIS models for synthesis of open supported coplanar...

[38] Guney K., Sarikaya N.: Adaptive-network-based fuzzy inference system models for narrow aperture dimension calculation of optimum gain pyramidal horns. Neural Network World, 8 , 2008, pp. 341-363.

[39] Yildiz C., Guney K., Turkmen M., Kaya S.: Adaptive neuro-fuzzy models for the quasi-static analysis of microstrip line. Microwave Opt. Technol. Lett., 50, 2008, pp. 1191-1196.

[40] Yildiz C., Guney K., Turkmen M., Kaya S.: Analysis of conductor-backed coplanar waveguides using adaptive-network-based fuzzy inference system models. Microwave Opt. Technol. Lett., 51, 2009, pp. 439-455.

[41] Turkmen M., Yildiz C., Guney K., Kaya S.: Adaptive-network-based fuzzy inference system models for air-suspended trapezoidal and rectangular shaped microshield lines. Microwave Opt. Technol. Lett., 52, 2010, pp. 20-24.

[42] Turkmen M., Yildiz C., Guney K., Kaya S., Anfis models for the quasi-static analysis of coplanar strip line structures. Microwave Opt. Technol. Lett., 52, 2010, pp. 1990-1996.

[43] Kaya S., Guney K., Yildiz C., Turkmen M.: Anfis models for synthesis of micro-coplanar stripline and asymmetric coplanar stripline with an infinitely wide strip. Microwave Opt. Technol. Lett., 54, 2012, pp. 460-467.

[44] Kirkpatrick S., Gelatt C. D. Jr., Vecchi M. P.: Optimization by simulated annealing. Science 220, 1983, pp. 671-680.

[45] Marquardt D. W.: An algorithm for least-squares estimation of nonlinear parameters. SIAM J. Appl. Math., 11, 1963, pp. 431-441.

[46] Dennis J. E.: Nonlinear Least-Squares, State of the Art in Numerical Analysis. Academic Press, 1977

[47] Holland J.: Adaptation in Natural and Artificial Systems. MI: University of Michigan Press, 1975.

[48] Goldberg D. E.: Genetic Algorithms in Search, Optimization and Machine Learning. Reading, Addison Wesley, MA, 1989.

[49] Zeland Software Inc., IE3D ${ }^{\mathrm{TM}}$, www.zeland.com, 2009. 